\title{
Entrelacs
}

Cinéma et audiovisuel

$N^{\circ} 17$ / Enjeux Audiovisuels du cinéma 360

\section{Un espace « Cannes XR » au Marché du Film :Quels enjeux pour l'industrie du cinéma?}

Hélène Laurichesse

\section{(2) OpenEdition \\ Journals}

\section{Electronic version}

URL: http://journals.openedition.org/entrelacs/5948

DOI: 10.4000/entrelacs.5948

ISSN: 2261-5482

Publisher

Éditions Téraèdre

\section{Electronic reference}

Hélène Laurichesse, « Un espace « Cannes XR » au Marché du Film :Quels enjeux pour l'industrie du cinéma? », Entrelacs [Online], 17 | 2020, Online since 01 July 2020, connection on 03 July 2020. URL http://journals.openedition.org/entrelacs/5948; DOI : https://doi.org/10.4000/entrelacs.5948

This text was automatically generated on 3 July 2020 .

Tous droits réservés 


\title{
Un espace « Cannes XR » au Marché du Film :Quels enjeux pour l'industrie du cinéma?
}

\author{
Hélène Laurichesse
}

À quoi ressemble l'avenir du film? Ça commence au Marché du film où chaque année, des professionnels du monde entier se réunissent pour partager et découvrir les dernières tendances de la production et de la distribution cinématographique ${ }^{1}$.

Moins anecdotique qu'il n'y paraît, cette publication, en entremêlant le devenir du cinéma à celui du marché du film à Cannes (MIF) pointe l'importance de ce dernier pour témoigner des tendances de l'industrie du cinéma. Aussi, lorsqu'en 2019, le MIF introduit un nouvel espace baptisé "Cannes XR", offrant ainsi une visibilité appréciable aux technologies immersives, ce parti pris est d'autant plus notable qu'il s'inscrit dans le contexte symbolique et polémique d'un festival de Cannes défendant une vision traditionnelle du film avec l'exigence (désormais isolée) que tous les films en compétition sortent obligatoirement en salles ${ }^{2}$. Certes, les objectifs du marché et du Festival de Cannes peuvent présenter des différences, mais leur imbrication, nous le verrons, ne s'est jamais démentie tout au long des dernières décennies. Bien au contraire, ils fonctionnent à la manière d'un couple, se renforçant l'un et l'autre de façon complémentaire, un pied dans l'artistique, l'autre dans l'économique. L'espace "Cannes VR ", évoque pourtant la perspective d'un cinéma qui se situe au-delà de son dispositif historique réunissant un public dans une salle devant un écran. "C'est parce que la nature de l'art a changé que du cinéma devient pensable en dehors d'une histoire qui s'est plus ou moins confondue avec l'exception de son dispositif historique ${ }^{3}$ " souligne Luc Vancheri en s'inscrivant dans la lignée des récents travaux académiques sur le devenir du cinéma ${ }^{4}$. Ces derniers tendent à l'optimisme pour considérer le rayonnement d'un cinéma qui ne saurait se confondre avec un dispositif technologique figé, bien au contraire, ce dernier étant amené à évoluer avec son contexte et à s'adapter. "S'adapter", c'est d'ailleurs le maître mot mobilisé par les personnalités hollywoodiennes interrogées sur la survie des films dans les dix prochaines années ${ }^{5}$. S'adapter à ce qu'Emmanuel Durand ${ }^{6}$ appelle "l'heure hybride ", celle des technologies, 
celle des pratiques des publics, celle d'une nouvelle cinéphilie ${ }^{7}$ dans un environnement numérique qui met en question la filière et le film depuis sa création en passant par sa production et ses circuits de distribution et de diffusion. C'est toutefois dès ses origines que le cinéma a été confronté au défi de ce renouvellement permanent, dans une dynamique d'inventions et d'innovations qui nécessitent pour être couronnées de succès une bonne connaissance du marché et de ses évolutions ${ }^{8}$. Les innovations apportées par les technologies immersives présentent à ce titre la potentialité d'apporter au secteur cinématographique un positionnement fortement différenciateur au regard des nouveaux usages des publics dans le contexte de développement de l'offre des plateformes. Une perspective porteuse qui explique très certainement la présence de la réalité étendue au sein du plus influent des marchés du film. En ce sens, l'étude de cas de «Cannes XR» au MIF nous semble susceptible d'apporter quelques éclairages sur les liens à établir entre les industries des technologies immersives et celle du cinéma. Comment le marché s'approprie-t-il ces technologies, avec quels objectifs, et quels moyens, et pour quel public? Ces questionnements s'inscrivent dans la lignée des travaux des Productions Studies ${ }^{9}$ qui visent à conceptualiser les pratiques de travail des professionnels en mettant l'accent sur le monde de la production envisagé comme une culture, avec ses propres codes et rituels. Après avoir défini ce que l'on entend ici par "marché » et par «XR ", nous évoquerons à travers "Cannes XR » comment l'industrie du cinéma s'empare des innovations des technologies immersives et quelles perspectives et limites se dessinent dans cette dynamique.

\section{$X R$ : Le champ des technologies immersives}

2 La terminologie de «XR » qui renvoie à la réalité étendue ${ }^{10}$ n'est pas encore usuelle dans le langage courant, elle correspond à l'évolution rapide des technologies dites immersives qui se déploient désormais au-delà de la réalité virtuelle (VR). Dans le secteur culturel, elles sont rattachées à ce que l'on appelle les " arts numériques ${ }^{11}$ » même si l'appellation ne fait pas tout à fait consensus, multimédia, transmédia, création numérique lui étant parfois préférée. La rencontre entre art et science qui caractérise ce secteur lui confère un positionnement atypique comparativement à celui des arts, mais aussi à celui des industries culturelles qui s'appuient sur des processus de création et des modèles économiques différents. La création dite numérique engendre en effet la mise en œuvre de collaborations complexes mêlant des créateurs, des ingénieurs, des scientifiques en posant une difficulté à identifier ce qui relève de l'artistique et ce qui relève de la technique. Elle s'exprime dans les performances audiovisuelles $(\mathrm{A} / \mathrm{V})$, les arts de la scène et en particulier la danse, la réalité virtuelle, le Net art, le Bio art, l'art robotique, le jeu vidéo, la stéréolithographie, la téléprésence ${ }^{12}$ pour faire émerger des œuvres par essence variées et hybrides qui viennent entrecroiser ces différents domaines. Elle recourt par ailleurs à des modalités spécifiques de financement, de diffusion, de commercialisation et de conservation et se distingue par ses dimensions interactive et immersive, deux concepts assez largement discutés dans les recherches académiques. Au sujet de l'œuvre immersive dans les arts numériques, les définitions proposées dans le dossier CRISP - bien qu'ouvrant très certainement au débat - nous semblent assez éclairantes sur les perspectives qu'elles impliquent pour pouvoir servir de référence à notre analyse :

Le propre d'une œuvre immersive, c'est de pouvoir faire vivre une expérience artistique, esthétique de l'intérieur, en réservant au spectateur la place centrale et 
non pas une confrontation classique avec une œuvre, en face à face, comme pour l'accrochage d'un tableau ou la visualisation sur écran.

[...] Au sens strict, les arts numériques sont à la source d'une véritable «interaction ", en sollicitant un mouvement de l'œuvre vers le spectateur et, inversement, du spectateur vers l'œuvre ${ }^{13}$.

3 Nous compléterons cette approche par celle envisagée dans le secteur audiovisuel avec une création numérique reprenant ces deux dimensions au prisme de l'œuvre audiovisuelle :

Par œuvre immersive et interactive, on entend des projets de création audiovisuelle, développant une proposition narrative, et destinés à un ou plusieurs supports qui permettent une expérience de visionnage dynamique fondée sur l'activation de contenus ou par simple déplacement du regard. Ce champ d'intervention recouvre notamment les œuvres destinées aux technologies immersives (réalité virtuelle et augmentée) et les narrations interactives conçues pour le web ou les écrans mobiles. Naturellement hybrides et transdisciplinaires, ces œuvres se situent principalement au point de rencontre du cinéma et du jeu vidéo ${ }^{14}$.

4 Cette approche, vouée à " révolutionner ${ }^{15}$ » l'expérience du public d'un film :

Consiste à faire entrer le spectateur dans une histoire, un jeu, une composition musicale, une œuvre d'art contemporain, de danse, de théâtre, en sollicitant plusieurs sens par des technologies comme la réalité virtuelle, la réalité augmentée, l'audio spatialisé, le vidéo mapping pour une expérience solo ou collective réalisée dans un espace public. Il peut s'agir d'un lieu dédié, orienté divertissement (LBE « Location Based Entertainment »), ou de lieux non dédiés comme des musées, salles de spectacles, centres d'art, etc ${ }^{16}$.

5 Avec l'expérience immersive, il est possible de vivre une réalité en $360^{\circ} \mathrm{en}$ regardant non plus seulement un écran devant soi, mais en percevant tout ce qui nous entoure avec une possibilité d'interagir. Pour parvenir à cette immersion, différentes technologies se combinent pour former l'ensemble vaste regroupé sous le terme générique de réalité étendue $(\mathrm{XR})$ désignant à la fois la réalité augmentée (AR) et la réalité virtuelle (VR).

6 De manière synthétique «la XR viendrait pallier le manque d'immersion de la réalité augmentée et la non-prise en compte du réel de la réalité virtuelle ${ }^{17}$ ». En l'occurrence, l'immersion par le biais de la VR se réalise avec un casque qui coupe de la réalité pendant que l'AR introduit des objets virtuels dans le champ de vision de l'utilisateur sur l'image du monde réel. Enfin ce que l'on appelle la réalité mixte (MR) combine objets virtuels et réels et se situant à mi-chemin entre une expérience de réalité virtuelle et de réalité augmentée.

\section{Art \& Industrie : Les marchés de l'économie de la culture}

7 Si la définition la plus large du marché peut le présenter comme une place d'échange, l'approche économique et l'approche sociologique n'y projettent pas les mêmes perspectives, respectivement transactionnelles et relationnelles. Du point de vue de l'économie, le marché est un point de rencontre entre l'offre et la demande qui permet la formation d'un prix dans le cadre d'une transaction (achat/vente). « Un lieu physique dans certains cas, mais surtout un lieu abstrait dans le cadre duquel sont atteints des points d'équilibre, en déterminant les quantités échangées et les prix de cession ${ }^{18}{ }$. Du point de vue 
de la sociologie, le marché représente un lieu dans lequel se construisent des relations sociales entre ses différents acteurs.

8 À ce titre, les marchés constituent des places d'observation privilégiées des évolutions et tendances d'une industrie, de leur dynamique créative et renseignent la connaissance et la compréhension du fonctionnement du secteur. Ils constituent aussi le lieu de découverte des talents si bien que leur rapport à la création, à la production et à la valorisation d'œuvres est de plus en plus questionné, comme l'illustrent deux questions essentielles posées dans l'éditorial de la revue Marges dans un numéro consacré à "L'art avec ou sans le marché »:

Le marché est-il réellement devenu le prescripteur principal des valeurs artistiques?

Le marché détermine-t-il l'évolution des productions artistiques? Et si c'est le cas, comment et par quels relais ? ${ }^{19}$

9 Le rôle du marché ne serait donc pas circonscrit à trouver des débouchés à des œuvres déjà produites, mais également à façonner, conditionner, accompagner, des œuvres en développement, voire à faire et défaire des « réputations $»^{20}$.

Dans le secteur artistique, il faut toutefois rappeler que le marché peut difficilement être appréhendé d'une façon homogène, au regard des différences observées dans les modalités de fonctionnement du secteur des arts et des industries culturelles. Si le travail créateur, le caractère aléatoire de la demande, l'exigence d'un renouvellement constant des produits, l'importance de la notoriété et des récompenses constituent des spécificités communes aux domaines artistiques, chaque marché présente ses caractéristiques propres. Le marché de l'art notamment fonctionne autour d'acteurs tels que les galeries, les critiques, les commissaires-priseurs, les conservateurs, les collectionneurs, les enseignants des écoles d'arts. La formation de la valeur et des prix s'y réalisant à partir de l'expertise scientifique de ces acteurs sur la base de la rareté des biens. Le marché des industries culturelles ${ }^{21}$ qui concerne le secteur du cinéma renvoie à des entreprises qui utilisent des méthodes industrielles pour la reproduction des œuvres culturelles (par exemple, le film œuvre unique se démultiplie avec les copies du film) à des fins d'exploitation commerciale en composant avec une dimension créative prédominante dans le processus de production et une dimension économique plus contraignante que dans le secteur des arts. Sur ce marché, le prix (le plus souvent encadré par les pouvoirs publics) n'étant pas un indicateur de qualité, la notoriété, et le système de médiation y est déterminant.

11 Le marché des arts numériques quant à lui est historiquement davantage associé au marché de l'art qu'à celui du film, tout en présentant de grandes spécificités :

Les artistes post-Internet - le terme ayant lui-même fait l'objet de nombreux débats

- sont considérés comme une génération qui pense avec et au travers des

technologies en réseaux, dans ses œuvres mêmes ${ }^{22}$.

12 La proximité de ses créations artistiques avec celles du design et avec celles du monde du spectacle le marginalise dans le monde des arts et sa dimension expérimentale reposant sur des œuvres rarement commercialisables le rend atypique dans le secteur des industries culturelles. On pourra ainsi étendre la réflexion posée comme suit à propos du secteur de l'art à celui des industries culturelles :

[...] l'interdisciplinarité des arts numériques entre en conflit avec les catégories éprouvées de l'art contemporain, qui ont été construites au fil des années par des spécialistes qui souhaitent garder leurs champs d'intervention ${ }^{23}$. 
Dans cette perspective, il convient de garder à l'esprit une spécificité des technologies immersives dans leur croisement entre différentes disciplines artistiques, le cinéma, le théâtre, la danse, le jeu vidéo pour questionner le rapprochement entre le marché du film de Cannes et le secteur des technologies immersives.

\section{Cannes XR}

14 L'espace "Cannes XR », initié en 2019, a été introduit dans le cadre du marché international du film inauguré en 1959, soit, treize ans après le Festival de Cannes. Le MIF organisé de manière très artisanale et improvisée lors des premières années s'est ensuite structuré au fil des décennies pour devenir un rendez-vous « incontournable ${ }^{24}$ » de tous les professionnels de l'audiovisuel. La grande majorité des sociétés y réalisant leur chiffre d'affaires de l'année, une absence à Cannes est généralement interprétée comme un signal de mauvaise santé d'une société audiovisuelle ${ }^{25}$. En 1995, sous la direction de Jérôme Paillard, le festival et le marché deviennent imbriqués dans leur fonctionnement, le MIF intégrant l'association française du festival. Ce faisant, l'évènement cannois devient pionnier d'une forme d'industrialisation des festivals qui va se généraliser dans la profession (Toronto, Berlin, Venise notamment). Une évolution qui correspond à ce que Dina Iordanova ${ }^{26}$ appelle " Festival Industry Oriented " en observant que le rôle initial du festival qui se situait traditionnellement au niveau d'un soutien à l'activité de distribution est en train d'évoluer sur le terrain du développement et de la production en prenant exemple sur Cannes et sa combinaison gagnante « marché-festival ».

La double visée culturelle et commerciale qui caractérise l'évènement cannois se traduit par une très forte imbrication des deux évènements :

Dans une manifestation bien organisée comme celle du festival de Cannes, personne n'est capable de distinguer ce qui relève du marché et ce qui relève de l'évaluation artistique, quels acteurs participent à l'une ou à l'autre des différents systèmes de légitimité et de valorisation ${ }^{27}$.

Dès lors, on peut observer une évolution de la fonction des «festivals » vers une dimension créative, y compris en l'absence de marché officiel. Devenus des «managers créatifs » et des "intermédiaires culturels », ils permettent aux films d'exister dans leur développement et leur production ${ }^{28}$. L'exemple cannois, emblématique de ce positionnement double, confirme son importance en tant qu'évènement référent pour l'ensemble du réseau des festivals.

Considérons à présent l'espace «Cannes XR » au sein du MIF en rappelant le rôle d'accompagnement des professionnels dans leurs démarches transactionnelles et relationnelles dont il s'est investi. Des stands, des salles de projection et des espaces de conférences et travail les y accueillent dans un espace découpé en différentes zones (Illustration 1) avec un accès réservé aux professionnels accrédités ${ }^{29}$. L'espace intérieur "Riviera», qui regroupe les stands et pavillons des exposants professionnels de différents pays et secteurs, accessibles par l'entrée principale du Palais des festivals au niveau - 1 (Illustration 2) est celui qui a hébergé en 2019 « Cannes XR ». Il est complété des espaces pour les exposants institutionnels et les pays à l'extérieur avec les zones Pantiero et Riviera. 


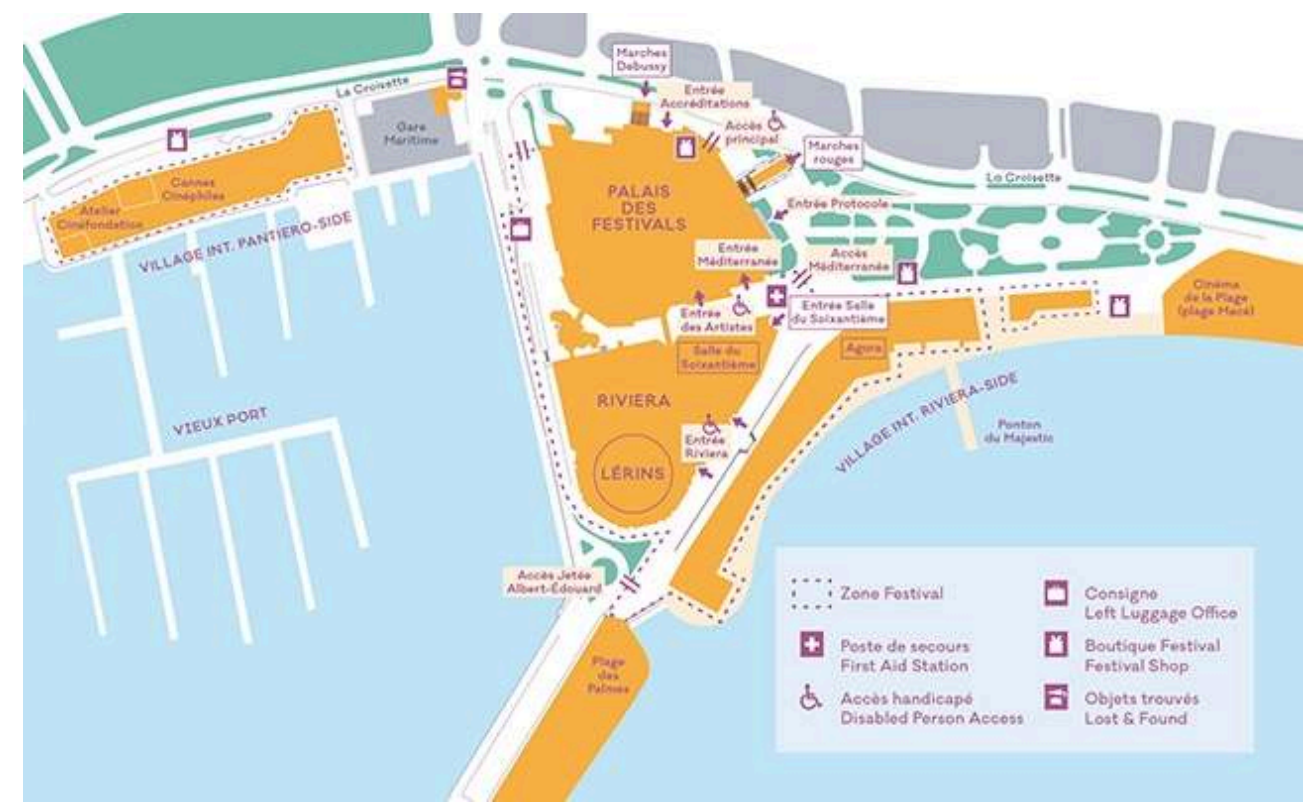

Illustration 1 : Le FIF et le MIF à Cannes. Source Site officiel du Festival

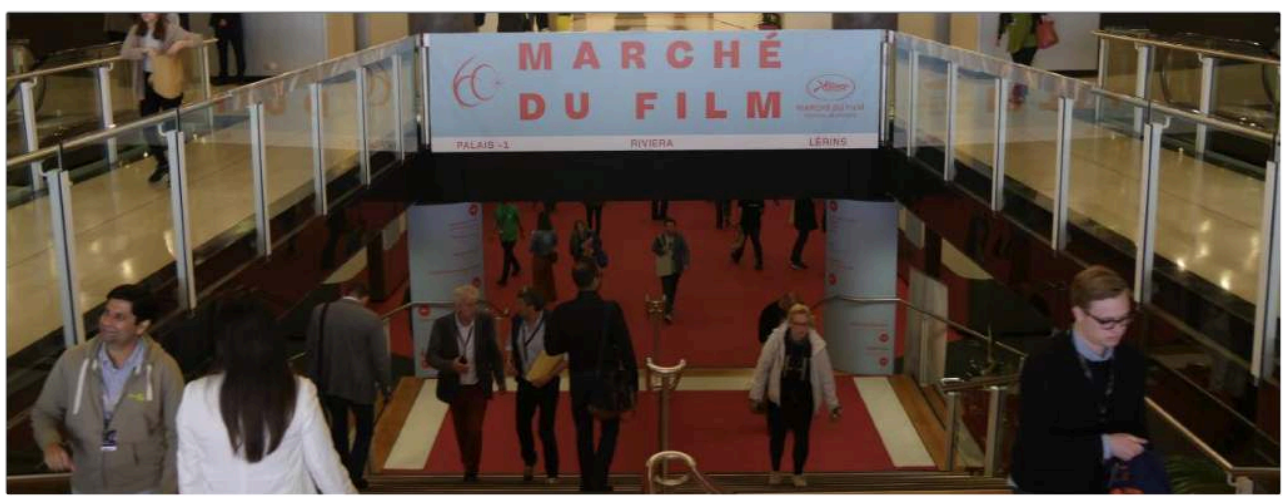

Illustration 2 : Espace Riviera au marché du film. Source : Photo personnelle

Le MIF, reconnu comme le plus grand marché du cinéma sur un plan international, regroupe 12500 professionnels, 4000 films et projets, 1400 projections, 121 pays $^{30}$ et représente 1 milliard de $\$$ de chiffre d'affaires généré en $2019^{31}$. Les activités s'y organisent autour de deux axes principaux :

- Le premier qui représente la plus grosse partie du chiffre d'affaires concerne désormais les projets. Les activités sont consacrées à la recherche de partenaires financiers, artistiques ou techniques sur la base de séances de pitching organisées pour trouver des producteurs ou de sélections dans des résidences d'écriture ou autres workshops ${ }^{32}$. À cet effet, le travail peut être initié très en amont à travers notamment la plateforme Cinanado ${ }^{33}$ qui recense tous les professionnels présents et les dates de leur présence. Il peut également s'appuyer depuis 2016 sur application dédiée à l'envoi d'invitations et au visionnage offline Service Match \& Meet.

- Le second est relatif aux films terminés pour lesquels des achats et ventes vont être effectués avec des chiffres stables autour d'un peu moins de 3000 films en ventes et 1400 projections. À la façon d'un zapping, les acheteurs passent d'une salle de projection à l'autre et s'ils sont intéressés par un film, se rendent ensuite sur les stands pour négocier le meilleur prix. 
19 L'imbrication «festival-marché » permet aux professionnels de mener de front plusieurs activités en même temps, ils viennent à la fois défendre un film en compétition dans une des sélections, vendre ou acheter les films produits pour le marché national ou international ${ }^{34}$, pré-vendre leurs nouveaux projets, sceller des partenariats.

20 Au sein du bâtiment Riviera, l'espace « Cannes XR » se situe au côté de lieux dédiés à la fiction (projections des films, stands des pays et des distributeurs du monde entier, au documentaire (Cannes Docs), au film court (Short Film Corner), à l'animation (Animation Day), aux nouvelles technologies (Next). L'espace « Next», initié en 2017, était supposé représenter jusqu'en 2019 l'ensemble du secteur des technologies, mais la VR y occupant $90 \%$ des activités représentées en 2018, la décision de créer un espace spécifique pour les technologies immersives a été prise. "Next » se consacre à présent à la valorisation de l'ensemble des nouveaux modèles d'innovation autour des blockchains, de l'intelligence artificielle, des start-up, des nouveaux concepteurs.

21 Pour l'organisation de l'espace XR, une carte blanche a été donnée à Elie Levasseur ${ }^{35}$, un Français basé à Londres spécialisé dans le secteur de l'industrie VR. Selon ses dires, l'orientation retenue pour cette inauguration était celle d'un positionnement B2B destiné à favoriser les rencontres entre professionnels du secteur. La promotion du secteur XR auprès des acteurs traditionnels de l'industrie cinématographique constituait l'objectif premier avec l'idée que l'émulation autour de cette nouveauté à Cannes pourrait attirer certains festivaliers venus pour le cinéma. Pendant six jours (du 14 au 19 mai 2019), environ 1000m2 ont été dédiés à "Cannes XR » dans les $10000 \mathrm{~m} 2$ du bâtiment Riviera, composé d'une salle de conférence de 80 places, d'un salon-vitrine VIP pour présenter les nouveaux projets, d'une arcade VR avec 10 stations proposant une cinquantaine de films, et d'une douzaine de stands de sociétés spécialisées dans la technologie $\mathrm{XR}^{36}$. Une extension de cet espace était prévue pour l'année $2020^{37}$ dans le site prestigieux de l'ancien casino Palm Beach, (1600m2) avec de nouvelles propositions (une compétition pour la meilleure œuvre cinématique XR notamment). 


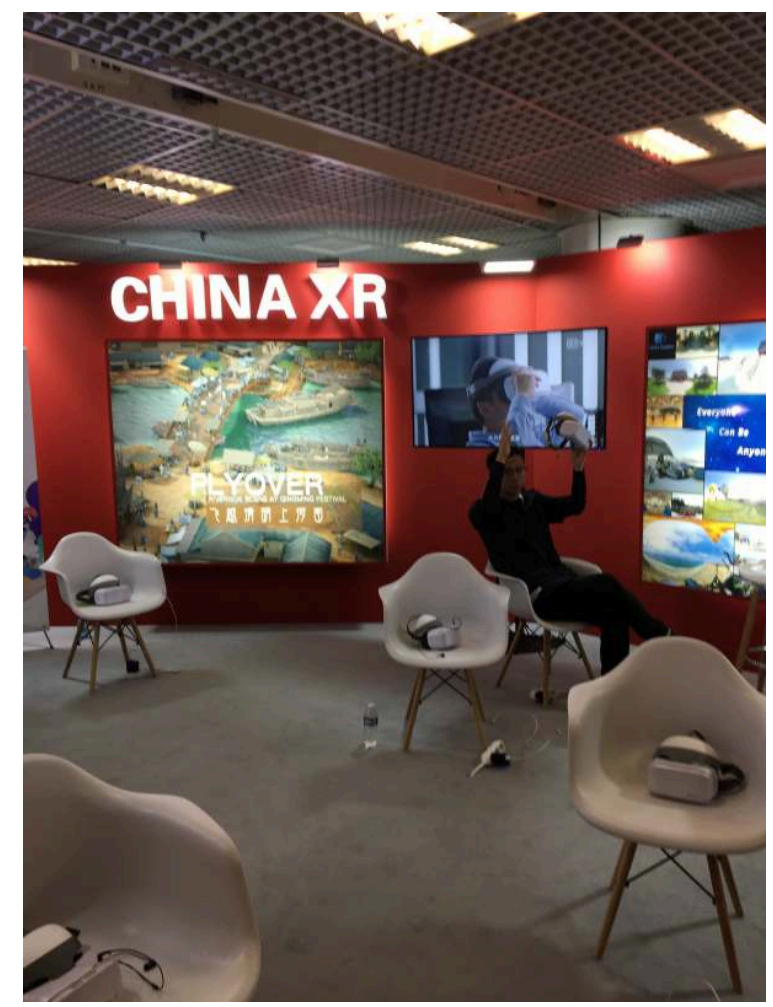

Illustration 3 : Stand chinois dans l'espace Cannes XR. Source : Photo personnelle

Du point de vue du marché, la mise en relation entre le cinéma et la XR, nous semble se formaliser à deux niveaux, le niveau du film et des pratiques de création, de production et de distribution et le niveau de l'industrie du cinéma avec la dimension encore expérimentale du secteur XR.

\section{Quand les contenus rencontrent les technologies immersives}

\section{WHERE STORYTELLING} MEETS IMMERSIVE TECHNOLOGIES

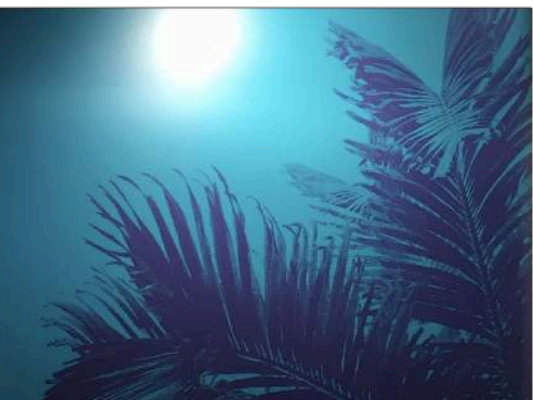

\section{\#cannesXR}

Illustration 4 : Quand la narration rencontre les technologies immersives. Source : Visuel officiel Cannes XR

Le visuel de présentation de l'espace «Cannes XR ( (Illustration 4) met l'accent sur la rencontre entre la narration et les technologies. Le défi de ce croisement est de parvenir à articuler ces deux ressources afin de créer de la valeur pour la filière cinématographique. Pour ce faire, expérimenter la maîtrise d'une "écriture 
immersive » constitue le premier des enjeux à relever. Oriane Morriet ${ }^{38}$ démontre à cet effet la nécessité pour les scénaristes ayant une expérience du cinéma à intégrer des aptitudes et compétences spécifiques pour une expérience audiovisuelle visant à proposer une dimension spatiale et interactive. Il leur faudra notamment acquérir des connaissances qui jusqu'alors ne relevaient pas de leur métier, en l'occurrence, celles relatives aux conditions de production et de diffusion. Apprendre à écrire un scénario en anglais devient aussi un impératif dans un contexte de production internationalisée. La faisabilité des œuvres qui mobilisent les technologies XR est conditionnée par des impératifs financiers et techniques, plus encore que dans la fiction traditionnelle, même si le point d'entrée (voulu par le MIF tout au moins) dans l'univers XR privilégie le récit. Autrement dit, la technologie vient en appui du récit, mais le récit doit nécessairement intégrer les potentialités la technologie. Il en va de même pour la connaissance du contexte de diffusion des œuvres, en raison des investissements très lourds qu'impliquent les dispositifs d'exposition, les scénaristes doivent être en mesure de choisir des options de récit qui pourront faciliter la diffusion dans des lieux dédiés ou via une plateforme. Il est à noter que le format court des œuvres en VR s'explique notamment par ces contraintes. Il faudra aussi que les scénaristes se familiarisent avec de nouvelles techniques de scénarisation mobilisant le «je » lors de l'écriture pour se projeter dans "l'expérience utilisateur", dans la perspective de limiter les problématiques physiques (motion sickness). L'utilisation de documents inédits empruntés aux domaines de la chorégraphie, des jeux vidéo et du théâtre permettront de « spacialiser » l'écriture avec des plans au sol ou des story-boards à cadrans.

L'écriture n'est naturellement pas la seule étape qui doit apprendre à composer avec ce nouveau langage, les réalisateurs de cinéma n'étant pas formés pour la majorité pour utiliser les caméras $360^{\circ}$, la notion de cadre est complexe à gérer dans une expérience où le spectateur peut regarder là où il veut. Il faut donc apprendre à travailler des ressorts scénaristiques et de mise en scène, pour essayer de contrôler son expérience et avec elle l'histoire qui est racontée, à la façon d'un tour de magie. Faire en sorte de provoquer une curiosité visuelle ou sonore par exemple pour attirer le regard à un endroit donné constitue un enjeu nouveau pour les scénaristes et réalisateurs ${ }^{39}$. Le mode d'expression de la VR puisant dans la chorégraphie, le théâtre, le jeu vidéo, c'est tout un nouvel arsenal de connaissances et de compétences auxquels doivent s'ouvrir les créatifs dans le secteur $\mathrm{VR} / \mathrm{XR}^{40}$. Les aptitudes requises jusqu'alors pour exercer leur métier s'en trouvent modifiées, nécessitant une ouverture plus large à la connaissance des modalités de travail ayant cours dans d'autres secteurs artistiques.

Dans le souhait de présenter les innovations technologiques à travers des contenus, l'espace XR au MIF proposait un espace de visionnage en partenariat avec la société SpringboardVR à partir d'un catalogue de films en VR disponible sur des bornes telles que présentées sur l'illustration 5 au moyen de casques à disposition. 


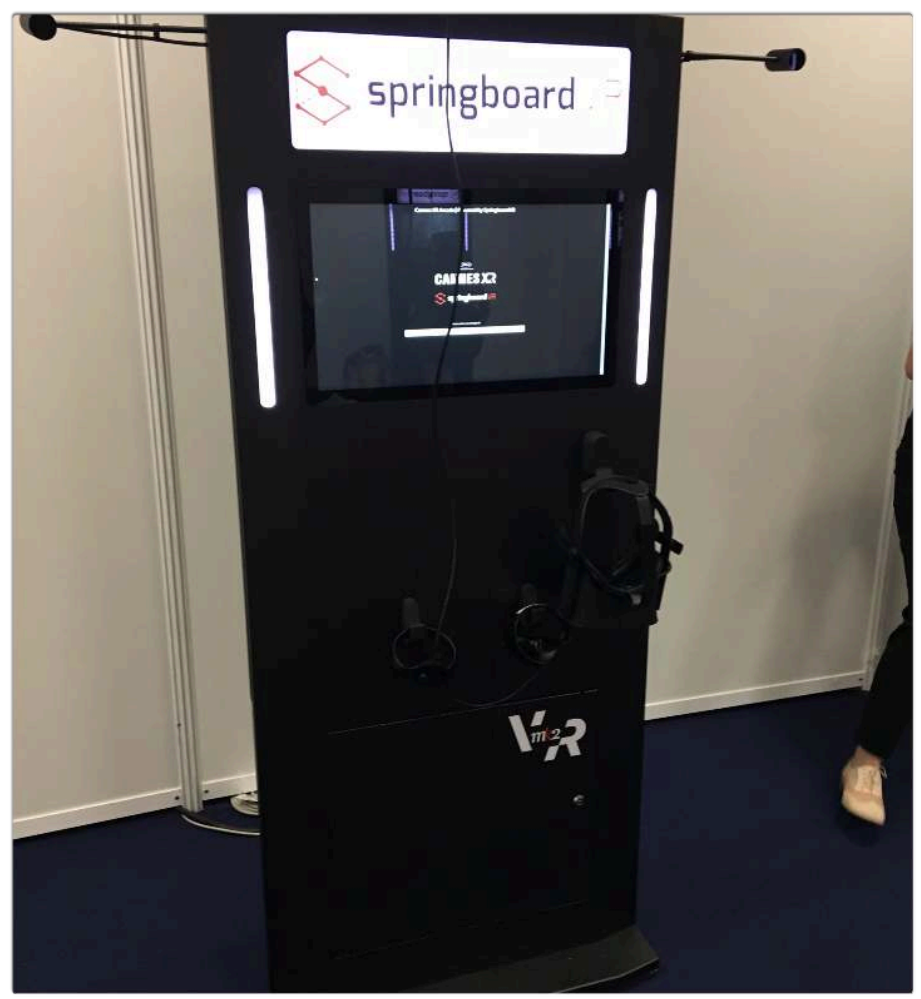

Illustration 5 : Bornes de visionnage de l'espace XR au MIF. Source : Photo personnelle

D'autres expériences immersives pouvaient être testées sur les stands ou lors de showcases. À titre d'exemples on peut citer une séquence immersive de la chanson " You're The One That I Want » du film culte Grease proposée par les sociétés Intel et Paramount expérimentant la capture 3D volumétrique, une expérience des premiers pas sur la lune proposée par Universal Pictures, les premières images en avantpremières des créations de Jan Kounen (7 Lives et Ayahuasca (Kosmik journey). Parallèlement aux démonstrations, des séances de workshops et pitchings se tenaient dans l'espace, une nouveauté était programmée pour l'édition 2020 avec un concours organisé par la société Positron, sponsor de l'évènement avec un prix de $60000 €$ de prix aux contenus primés.

Selon notre expérience des œuvres visionnées sur place, cette rencontre entre la narration et la technologie reste encore superficielle, la majorité des œuvres présentées faisant davantage appel à la sensation qu'à la narration. Cette dernière, souvent peu construite, apparaît plus comme un prétexte à la démonstration technologique que comme un véritable enjeu en dépit du discours autour des ambitions narratives. Une problématique d'ailleurs abordée de front lors de l'évènement «Cannes XR » avec une conférence spécifique à la problématique de la narration destinée à réfléchir à ces questions avec les différents professionnels de la filière du cinéma.

\section{Des technologies immersives innovantes pour le cinéma}

"L'industrie cinématographique est la plus technologique des industries créatives ${ }^{41}$ » soulignent Elisa Salvador, Jean-Paul Simon, Pierre-Jean Benghozi en rappelant sa longue histoire de nouveaux entrants et nouvelles technologies. Comme tout domaine 
artistique, le secteur cinématographique repose sur l'innovation, «la capacité (et la nécessité) de continuer à offrir aux consommateurs de nouveaux produits "créatifs" ». Son industrie s'est ainsi régulièrement renouvelée pour tenir compte d'un contexte environnemental en mutation constante (la télévision, la vidéo, Internet, le numérique). Autrement dit, les nouvelles technologies sont pour le cinéma à la fois de nouvelles sources d'inspiration pour ses auteurs et de nouvelles formes de concurrence pour son industrie. Le point de rencontre entre ces différentes évolutions se situe dans la nécessité de repenser l'expérience cinématographique et son modèle. Aujourd'hui, face à la concurrence des plateformes qui proposent une consommation de contenus instantanée, accessible à tout moment sur tablette, smartphone, ordinateur ou sur l'écran de la télévision, l'enjeu est de faire de la sortie en salle une expérience forte et mémorable en utilisant toutes les innovations technologiques disponibles. En ce sens, les technologies immersives présentent un attrait incontestable pour proposer une offre véritablement différenciée de celle de la consommation à domicile ou mobile. La dynamique d'innovation qui sous-tend le secteur XR requiert toutefois des investissements lourds dans la recherche que peut difficilement supporter l'industrie du cinéma. Les investissements se réalisent alors le plus souvent en dehors du circuit traditionnel et de la chaîne de valorisation d'un projet audiovisuel par des sociétés extérieures à ce secteur. On observe toutefois une évolution avec une implication des majors de l'industrie américaine dans la production des projets VR ambitieux, qui ne sont pas directement commercialisables, comme ceux présentés par les sociétés Paramount ou Universal dans l'espace Cannes XR. Le fonctionnement des majors désormais adossé à la gestion de propriétés intellectuelles, formalisées par des licences dans le cadre des franchises/marques (Stars Wars, Batman, etc.), favorise un engagement financier de la sorte au contraire des entreprises françaises qui n'ont pas cette culture de la licence.

Parmi ces innovations, les principales technologies présentées en 2019 à Cannes XR comme susceptibles de servir le cinéma concernent trois principales perspectives :

- la capture du mouvement des yeux afin de reproduire la vision naturelle et augmenter la qualité de l'image transmise sur les écrans pour enlever les sensations de cinétose.

- la capture volumétrique permettant de capturer le mouvement en 3D

- la $5 \mathrm{G}$ qui doit permettre l'allègement des dispositifs (câble notamment) en révolutionnant complètement l'écosystème de la XR

Force est de constater lorsqu'on découvre l'espace XR à Cannes, que la rencontre entre les deux secteurs se situe à un stade encore très expérimental. Le marché physique du MIF, est en ce sens important pour permettre aux entreprises spécialisées dans le secteur XR de présenter leurs innovations aux professionnels du cinéma et de l'audiovisuel de dédier dans un espace spécifique. La nature expérimentale de ce marché appliquée au cinéma permet de comprendre que les expositions s'adressent à des professionnels plus à même de juger le potentiel des projets à partir d'œuvres qui ne sont pas encore abouties sur un plan cinématographique en raison d'une narration et d'une mise en scène quelque peu défaillantes. On retrouve ici une problématique qui se pose aussi dans l'industrie vidéoludique :

Dès lors, un clivage s'affirme entre défenseurs d'une activité ludique qui ne requiert aucun paravent narratif pour exercer sa puissance d'attraction, et ceux pour qui la plasticité du jeu rend possible une nouvelle forme de cinéma, de jeu avec une histoire, une narration renouvelée, dynamique et en temps réel ${ }^{42}$. 
31 familiarisent avec les potentialités de la VR, que les technologies se stabilisent pour procurer une expérience compatible avec un bien-être physique, et tout autant pour trouver un modèle économique viable. C'est donc la dimension relationnelle du marché qui est envisagée dans le cadre du MIF pour une mise en relation des professionnels entre eux, rejoignant ainsi le rôle d'intermédiaires culturels des festivals et marchés évoqués précédemment. Pour autant, le MIF se positionne aussi dans une perspective de réflexion sur le modèle économique du couple Cinéma-XR. Vingt-quatre conférences ont été organisées à cet effet par le marché, sur les enjeux des LBE, les modalités de financement des œuvres, et en particulier les coproductions internationales, les enjeux de la $5 \mathrm{G}$, les aspects juridiques de la création immersive, le modèle économique de diffusion à l'international. Cette dernière conférence, présentée au pavillon les cinémas du monde, invitait les professionnels du secteur des réalités immersives à dialoguer avec les attachés audiovisuels du réseau culturel français à l'étranger afin de réfléchir à des modèles d'accompagnement vertueux de diffusion des créations immersives à l'étranger.

préhender du point de vue du marché le secteur XR est complexe en raison de son interdisciplinarité. Pour cette raison, des chiffres très différents peuvent être avancés selon que l'on considère ce qui relève des contenus ou ce qui relève de la recherche sur la technologie. Tous secteurs d'applications et technologies confondus ${ }^{43}$, le marché XR devrait atteindre 150 milliards de dollars en $2020^{44}$, se répartissant entre l'AR (120 milliards de dollars) et la VR (30 milliards de dollars). Les investissements dans ces technologies sont massifs avec la perspective que la XR devienne un marché pour le grand public de la même ampleur que celui du téléphone mobile ${ }^{45}$. Toutefois, dans ce panorama des technologies immersives, il est utile de rappeler que le secteur culturel ne représente qu'une faible partie des applications avec en tête le marché du jeu vidéo (5 milliards de dollars).

secteur artistique notamment où l'économie fonctionne sur des projets, ce sont les investissements dans les contenus qui priment avec un marché encore embryonnaire sur un plan strictement économique. Le chiffre d'affaires des films VR en France en 2019, autour de 70000 euros est encore faible en raison de l'absence de sociétés investissant dans le secteur. Arte qui représente une des sociétés les plus actives sur le secteur investit autour de 200000 euros par an (4 projets à 50000 euros) ${ }^{46}$. On notera toutefois la présence des sociétés Atlas $\mathrm{V}$ et Novelab dans le programme en trois volets Sphères en coproduction avec les sociétés américaines Protozoa Pictures et Crimes of Curiosity dans un accord d'acquisition à sept chiffres avec le soutien d'Oculus et d'Intel ${ }^{47}$.

Signe que l'industrie du cinéma affirme toutefois une volonté de s'impliquer dans le secteur, en France, le CNC a soutenu une centaine de projets depuis 2015. Initialement portées par le Fonds d'aide aux nouveaux médias, les aides le sont aujourd'hui par le Fonds d'aide aux expériences numériques (dénommé XN) qui le remplace. Ce fonds de $3 \mathrm{M} €$ en $2019^{48}$ intervient depuis l'écriture jusqu'à la production, il est géré par le service de la création numérique qui comprend les aides au jeu vidéo et les aides aux arts numériques. La production des films VR en France est conditionnée à ces soutiens publics via l'activité de ce fonds. 

celle des espaces physiques de type LBE, lieux intermédiaires ayant les moyens d'investir pour aménager des espaces permettant au public de venir vivre des expériences XR et celle de la consommation à domicile via des plateformes et des casques. La première option semble actuellement peu favorable suite à des fermetures de LBE qui tentent à montrer que le modèle n'est pas viable économiquement dans le cadre restreint de l'univers cinématographique. Le MK2 VR qui avait ouvert un espace permanent dédié à la VR à Paris en 2016 avec une douzaine de stations a récemment fermé, de même les salles IMAX ont également fermé leurs espaces VR en 2019 envoyant un signal très certainement désastreux pour le marché. L'espace Illucity à la Villette à Paris, qui se présente comme un parc d'attractions VR de type Escape Game plus ouvert sur le divertissement large semble mieux résister. Il reste alors en premier lieu les festivals et les marchés artistiques pour permettre ponctuellement de découvrir ces œuvres, comme dans le cas du MIF. Quant à la consommation à domicile, elle pose le problème de l'investissement dans des casques qui ont de fortes chances d'être dépassés technologiquement au bout d'un an, ce qui explique la faible progression de ce marché. Le marché domestique se situe par ailleurs hors du domaine marchand, la grande majorité des films étant disponibles gratuitement sur les plateformes telles qu'Oculus Store ou Steam par exemple.

modèle économique du cinéma XR renvoie naturellement à celle du public susceptible d'être intéressé par ces œuvres dans un contexte économique marchand. Sur cette question, une étude menée par le $\mathrm{CNC}^{49}$ vient confirmer que les usages du public en matière de VR concernent avant tout le jeu vidéo et les parcs d'attractions. On y apprend que $41 \%$ des Français ont déjà eu une expérience VR principalement dans ces domaines et pour la majorité il s'agit sans trop de surprise d'un public jeune. Sans œuvre de référence permettant de développer la notoriété du secteur (sur le modèle du film Avatar pour la 3D), et faute de lieux grands publics permettant la consommation de contenus VR, le marché grand public n'existe pas. L'espace XR à Cannes, accessible aux porteurs d'un badge professionnel Festival ou Marché ${ }^{50}$ n'est d'ailleurs pas ouvert au grand public ni même aux festivaliers cinéphiles qui ne sont pas des professionnels ${ }^{51}$. Comme il a été souligné, l'objectif du MIF à cette étape d'expérimentation est de sensibiliser les professionnels du cinéma aux technologies XR et les activités qui sont proposées dans l'espace sont en conformité avec cette ambition. Quant au public de «niche » supposé acquis des «gamers ", il offre des débouchés sans doute limités, les marchés de l'art et du divertissement présentant de grandes différences dans les motivations à l'origine des choix de consommation. Ce modèle non marchand d'exposition qui domine actuellement doit néanmoins évoluer dans vers un modèle marchand pour pouvoir poursuivre son expansion.

\section{Conclusion}

L'étude de l'espace «Cannes XR » introduit dans le marché du film en 2019, permet un double constat, d'une part sur la nature encore expérimentale de la rencontre entre la XR et le cinéma qui rend difficile le déploiement d'un marché tourné vers le grand 
public, d'autre part sur la fonction d'intermédiation du MIF qui vise à offrir le cadre et les conditions d'un développement des échanges entre les professionnels. La difficulté à envisager un modèle économique viable pour ce marché du cinéma XR dans sa phase expérimentale de tests se voit fragilisée par le retrait de certains acteurs phares du secteur et risque de se voir aggravée très certainement par le contexte de crise sanitaire de 2020. Les problèmes d'hygiène des dispositifs permettant ces expériences dans des espaces collectifs, jusqu'alors encore peu présents dans les enjeux de développement ${ }^{52}$, risquent de devenir prégnants dans la période à venir. La tendance à une consommation individualisée notamment à partir des smartphones (en hausse de $60 \%$ sur un an) a toutes les chances de se renforcer dans ce contexte y compris pour secteur XR. Jeffrey Katzenberg, créateur du service Quibi lancée le 6 avril aux ÉtatsUnis, disponible uniquement sur les téléphones portables ou les tablettes estime qu'il s'agit de « la troisième révolution de la narration ».

\section{NOTES}

1. Publication du compte Instagram du Marché du Film de Cannes 11 mars 2020

2. Une décision qui écarte ainsi les films de la plateforme Netflix, au contraire des Festivals de Venise ou Berlin où ils sont désormais acceptés.

3. Luc Vancheri, «Le cinéma après l'époque du cinéma » in Maxime Scheinfeigel, Le cinéma, et après ? Rennes : Presses universitaires de Rennes, 2010, p. 19-24.

4. André Gaudreault, Philippe Marion, La fin du cinéma? Un média en crise à l'ère du numérique, Paris, Armand Colin, 2013, Maxime Scheinfeigel, Le cinéma, et après? Rennes, Presses universitaires de Rennes, 2010.

5. Kyle Buchanan, «How Will The Movies (As We Know Them) Survive the Next 10 Years? ", The New York Times, 20/06/2019.[en ligne] https://www.nytimes.com/interactive/2019/06/20/ movies/movie-industry-future.html

6. Emmanuel Durand, La menace fantôme. Les industries culturelles face au numérique, Presses de Sciences Po, 2014, p. 9-16.

7. Jean-Paul Aubert, Christel Taillibert, Les nouvelles pratiques cinéphiles, Cahiers de Champs Visuels, n 12-13, Paris, Éditions l'Harmattan, Paris, 2015, 316 p ; Aubert Jean-Paul, Christel Taillibert, L'économie de la cinéphilie contemporaine, Cahiers de champs visuels $\mathrm{n}^{\circ} 14-15$, paris, Éditions l'Harmattan, 2017, $192 \mathrm{p}$; Michaël Bourgatte, Vincent Thabourey (dir.), Le cinéma à l'heure du numérique. Pratiques et publics, Paris, MkF Éd., collection Les Essais numériques, 2012, $223 \mathrm{p}$.

8. On pourra consulter à ce sujet l'histoire des différentes innovations technologiques qui a façonné le cinéma au fil des décennies dans le Chapitre 2 « Cinéma et Innovation » de l'ouvrage : Creton Laurent, Économie du cinéma, Armand Colin, Paris, $20145^{\mathrm{e}}$ édition, p 37-56.

9. Vicki Mayer, Miranda J. Banks et John Thornton Caldwell, Production Studies, New York, Routledge, 2009.

10. « Extended Reality ».

11. Laurent DIOUF, Anne VINCENT, et Anne-Cécile WORMS, «Les arts numériques », Dossiers du CRISP, vol. 81, $\mathrm{n}^{\circ} 1,2013$, pp. 9-84. [en ligne] https://www.cairn.info/revue-dossiers-ducrisp-2013-1-page-9.htm 
12. Pour approfondir ces différents aspects de la typologie des arts numériques, on pourra consulter Laurent DIOUF, Anne VINCENT, et Anne-Cécile WORMS, ibid.

13. DIOUF L., VINCENT A., et WORMS A.C, ibid.

14. CNC «Fonds d'aide aux expériences numériques» [en ligne] https://www.cnc.fr/ professionnels/aides-et-financements/creation-numerique/fonds-daide-aux-experiencesnumeriques_191100

15. Une terminologie souvent mobilisée dans les médias, pour exemple : «La réalité virtuelle vat-elle révolutionner le cinéma?», [en ligne] https://www.bfmtv.com/mediaplayer/video/larealite-virtuelle-va-t-elle-revolutionner-le-cinema-1161271.html, « Comment la réalité virtuelle transforme le cinéma » [en ligne] https://www.realite-virtuelle.com/cinema-vr/

16. «Expériences immersives, des nouvelles pratiques culturelles dans l'espace public ", [en ligne] https://www.cnc.fr/creation-numerique/etudes-et-rapports/etudes-prospectives/ experiences-immersives-des-nouvelles-pratiques-culturelles-dans-lespace-public_978218

17. "Après la réalité virtuelle, voici venir la réalité étendue!", [en ligne] https://www. 360natives.com/realite-etendue-30032018/

18. Laurent Creton, L'économie du cinéma en 50 fiches, Paris, Armand Colin, 2016, $5^{\mathrm{e}}$ Édition, p. 13.

19. Jerôme Glicenstein, «Éditorial », Marges, $n^{\circ} 28$ « L'art avec (ou sans) le marché de l'art », 2019, p. 5-8

20. Brian Moeran and Jesper Strandgaard Pedersen, Negotiating values in the creative industries: fairs, festivals and competitive events, Cambridge University Press, 2011.

21. Les industries culturelles couvrent les champs suivants : cinéma, disque, édition, gravure, photographie d'art, presse, télévision et nouveaux produits audiovisuels.

22. Ashley Lee Wong, «Comment se confronter au marché : les nouveaux médias face au marché de l'art », Marges n 28, 2019. [En ligne], http://journals.openedition.org/marges/1845

23. Laurent DIOUF \& al., ibid.

24. C'est le qualificatif que l'on retrouve systématiquement dans tous les articles ou reportages médiatiques consacrés au MIF.

25. Se rendre à Cannes représente un investissement (jugé rentable) pour les professionnels, une société indépendante de films d'auteurs comme Memento Films de taille moyenne investit tous les ans autour de 18000 euros avec une équipe d'une dizaine de personnes sur place. Sophie Benamon, Emmanuel Cirrode, «Cinéma et paillettes : Tout savoir sur le business du festival de Cannes ", L'express, 20/05/2012. [en ligne] https://www.lexpress.fr/culture/cinema/tout-sur-lebusiness-du-festival-de-cannes_1116074.html.

26. Dina Iordanova, «The Film Festival as an Industry Node », Media Industries Journal, vol. 1, n³, 2015. [en ligne]http://dx.doi.org/10.3998/mij.15031809.0001.302

27. Pierre-Jean Benghozi, Claire Nénert, "Création de valeur artistique ou économique : du Festival International du film de Cannes au marchẻ du film», Recherche et Applications en Marketing, Vol. 10, n 4, 1995, p. 65-76, Sage Publications. [en ligne] https://www.jstor.org/stable/ 40589022

28. Plusieurs chercheurs du Film Festival Research Network (FFRN) soulignent cette évolution. On peut retrouver quelques-unes de leurs contributions dans l'ouvrage collectif Marina De Valck, Brendan Kredell, Skadi Loist, (Dir.), Film Festivals,History, Theory, Method, Practice. Londres, Routledge, 2016, et sur le site Film Festival Research,. [en ligne] http:// www.filmfestivalresearch.org

29. Le public ne peut y accéder.

30. Pour les années 2017 et 2018 on peut consulter le détail des différents pays représentés au marché dans l'article Jérôme Paillard et Hélène Laurichesse, «Le marché du film du Festival de Cannes ", Entrelacs n 14, 2019. [En ligne], http://journals.openedition.org/entrelacs/4325

31. Ses principaux concurrents, ceux de Berlin (EFM) ou de Los Angeles (AFM), ne réunissant « que » 8000 participants à titre comparatif. 
32. Le Producers Network permet renforcer le réseau entre pairs, faciliter les coproductions internationales, Les Mixers sont des rencontres entre professionnels à la plage des palmes autour d'évènements spéciaux, Le Fantastic Fanatics Mixer rassemble toute la communauté du film fantastique. Le Doc Lovers Mixer permet aux professionnels du documentaire de se retrouver. Le Festivals \& Sales Agents Mixer organise la rencontre entre les programmateurs de festivals et les dirigeants de sociétés de vente. Lancement d'un programme format speed-meeting pour des rencontres avec les plateformes VOD à Cannes en 2019.

33. "Cinando" est la première base de données mondiale en ligne de l'industrie cinématographique, lancée en 2003. Elle offre des outils de mise en relation, un annuaire complet de sociétés et de personnes, des contacts, des films et projets en développement tout au long de l'année et lors des grands marchés internationaux. "Cinando Screeners » est une solution vidéo sécurisée pour facilement héberger et visionner des films en ligne.

34. Une grande diversité de genres de films est représentée au marché (contrairement au festival) où l'on trouve aussi bien des films d'auteurs indépendants que des films d'horreur ou «gore » de série $\mathrm{B}$.

35. Un entretien personnel a été réalisé auprès d'Elie Levasseur par nos soins

36. Les exposants 2019 comprenaient quatre sociétés françaises $\left(360^{\circ}\right.$ Film Festival, Onirix, Wide, VRROOM), quatre Américaines (Intel Corporation, Spherica, Kaleidoscope, Springboard), une Belge (VRTL), une Chinoise (Xinhuanet) une taiwanaise (Funique), une Polonaise (Immersify).

37. A l'heure où nous écrivons cet article, l'édition 2020 du festival et du MIF ont été reportés en raison de la crise sanitaire du Covid-19.

38. Oriane Morriet, «Concevoir et écrire pour la réalité virtuelle: nouvelles compétences, approches et techniques de scénarisation », Mise au point $n^{\circ} 12$, 2019. [En ligne] http:// journals.openedition.org/map/3825.

39. Le témoignage d'Antoine Cayrol à ce sujet est instructif. Lauret Jean-Kléber Okio Studios Producteurs de films en réalité virtuelle. Il y a 2 ans personne ne savait filmer en VR »6/12/2015 [en ligne] https://www.lesnumeriques.com/casque-realite-virtuelle/okio-studio-producteurfilms-en-realite-virtuelle-a2503.html

40. Un dossier présentant les métiers du cinéma, de la télévision, du jeu vidéo et de la création numérique est disponible sur le site du CNC. [en ligne] https://www.cnc.fr/cinema/dossiers/lesmetiers-du-cinema-de-la-television-du-jeu-video-et-de-la-creation-numerique_915361

41. Elisa Salvador, Jean-Paul Simon et Pierre-Jean Benghozi. «Facing disruption: the cinema value chain in the digital age ", International Journal of Arts Management, Vol. 22, n¹, 2019, p 15-40. 42. François Rouet, «La création dans l'industrie du jeu vidéo » janvier 2009. [en ligne] https:// www.culture.gouv.fr/Sites-thematiques/Etudes-et-statistiques/Publications/Collections-desynthese/Culture-etudes-2007-2019/La-Creation-dans-l-industrie-du-jeu-video-CE-2009-1

43. Secteurs d'applications principaux : le marketing (applications e-commerce), l'art, les réseaux (reconnaissance faciale), l'assistance = signaler danger sécurité, domaine médical, formation a certains métiers, familiariser travailleurs à des contextes atypiques

44. Étude du cabinet Digi Capital.

45. Pour exemple Facebook a investi 2 milliards \$ pour acquérir Occulus, Google 542 milliards \$ dans celle de Magic Leap.

46. Source : entretien avec Elie Levasseur

47. https://www.mediakwest.com/production/item/acquisition-record-par-citylights-pour-uneserie-vr-co-produite-par-atlas-v-pendant-le-festival-du-film-de-sundance.html

48. Sur un budget total de 803,5 $\mathrm{M} €$, la contribution reste toutefois timide.

49. «Réalité virtuelle et expériences immersives en France : quels usages ? [en ligne] https:// www.cnc.fr/creation-numerique/etudes-et-rapports/etudes-prospectives/realite-virtuelle-et-

experiences-immersives-en-france--quels-usages_978225 
50. Pour ce qui est des codes et protocoles de Cannes, on pourra consulter l'ouvrage d'Emmanuel Ethis. Emmanuel Ethis, Aux marches du palais. Le festival de Cannes sous le regard de sciences sociales, Ministère de la culture DEPS, 2001.

51. Inversement, des festivals dédiés à la VR existent (ex. Arles VR, Newimages) mais ce ne sont pas des festivals de films.

52. À noter toutefois qu'une conférence sur le sujet s'est tenue à Laval Virtual Word en 2019

\section{ABSTRACTS}

\section{Abstract}

In 2019, a new marketplace dedicated to immersive technologies was introduced at the Cannes Filmmarket, named « Cannes XR ». This article focuses on the collaboration between the both film and immersive technologies industries through this case study. We are studying the issues around technological frontier and its impact on the global film industry. We will see how the market can encourage and support collaboration between tech leaders and artists to contribute to the debat, networking, partnership and funding for immersive projects.

Résumé

En 2019, un nouvel espace dédié aux technologies immersives a été introduit au marché du film à Cannes baptisé «Cannes XR ». Nous nous intéressons dans cet article à ce rapprochement entre l'industrie du cinéma et celle des technologies immersives à travers cette étude de cas. Il s'agit de mettre en lumière les enjeux et problématiques autour des nouvelles technologies et de leur impact sur l'industrie du cinéma. Nous verrons comment le marché peut favoriser et accompagner les rencontres entre les professionnels pour susciter la réflexion, des opportunités de réseautage, de partenariat et de financement pour les projets immersifs.

\section{AUTHOR}

\section{HÉLÈNE LAURICHESSE}

Professeure à l'ENSAV (École Nationale Supérieure d'Audiovisuel) de l'Université Toulouse JeanJaurès. Membre du LARA-SEPPIA (Laboratoire en Recherche Audiovisuel-Savoirs, Praxis et Poḯtiques en Art), ses travaux portent sur la production, le processus de création, et les stratégies des industries culturelles. Elle a publié au CNRS Éditions «Quel marketing pour le cinéma ? » (2006) et « La stratégie de marque dans l'audiovisuel » (2013) aux éditions Armand Colin Recherche. Récemment, elle a dirigé le numéro 14 de la revue Entrelacs sur le thème « Marchés du film : évolutions, mutations et perspectives » (2018). 